\title{
Umbilical choline and related methylamines betaine and dimethylglycine in relation to birth weight
}

\author{
Marije Hogeveen ${ }^{1}$, Martin den Heijer ${ }^{2,3}$, Ben A. Semmekrot ${ }^{4}$, Jan M. Sporken ${ }^{5}$, Per M. Ueland ${ }^{6,7}$ and Henk J. Blom ${ }^{8}$
}

BACKGROUND: Low birth weight (LBW) is associated with increased morbidity and mortality for the newborn and risk of chronic disease in adulthood. Choline plays an essential role in the integrity of cell membranes, methylation reactions, and memory development. We examined whether choline, betaine, and dimethylglycine (DMG) concentrations were associated with LBW in Dutch women.

METHODS: Blood was sampled from umbilical cords (UCs) at delivery in singleton pregnancies $(n=1,126)$. Maternal blood was sampled at 30-34 wk of gestational age (GA) $(n=366)$. We calculated birth weights standardized for GA and defined LBW as standardized birth weight $\leq 2,500 \mathrm{~g}$.

RESULTS: Maternal concentrations were lower as compared with UC concentrations and were not associated with birth weight. UC choline and betaine were inversely associated with birth weight $(\beta=-60(-89,-31)$ and $\beta=-65(-94,-36)$, respectively), whereas UC DMG was positively associated with birth weight $(\beta=35(6.1,63))$. Odds ratios for LBW were 4.12 $(1.15,14.78), 5.68(1.24,25.91)$, and $0.48(0.09,2.65)$ for the highest UC choline, betaine, and DMG quartiles, respectively, as compared with the lowest quartiles.

CONCLUSION: We observed an increased risk of LBW with increased umbilical choline and betaine in venous UC blood. These results might reflect a change in choline consumption or metabolism or a disturbed placental function.

L

ow birth weight (LBW) poses the newborn an increased -risk of morbidity and mortality (1). LBW also relates to an increased risk of chronic disease in adulthood, e.g., hypertension, coronary heart disease, stroke, and diabetes (2), underlining the importance of revealing possible and moreover treatable risk factors for LBW.

Choline is an essential nutrient and is involved in several metabolic processes including lipid metabolism, methylation reactions, and synthesis of acetylcholine. It also plays a role in the structural integrity and signaling functions of cell membranes, brain development, and neurotransmission (Figure 1) $(3,4)$.

Pregnancy places a heavy burden on maternal choline stores, and choline is critical for the rapidly growing fetus $(5,6)$.
In utero, the fetus lives in a high-choline environment because of a system actively transporting choline against a concentration gradient across the placenta to the fetus $(7,8)$. Variation in maternal choline status could possibly influence fetal outcome through disturbances in cell membrane synthesis, increase in homocysteine concentrations, and/or perturbation of methylation reactions and changes in acetylcholine production. Choline also influences stem cell proliferation, apoptosis in the brain, and the developing hippocampus (memory center) in rodents (9). Maternal choline supplementation in rats during critical periods in pregnancy causes lifelong changes in brain structure (10). In addition, perinatal choline supplementation attenuates behavioral alterations associated with fetal or neonatal alcohol exposure in rats (11).

Clinically, low maternal choline concentrations or intake relate to neural tube defects $(12,13)$. Accumulation of choline in the fetal circulation has been observed by several authors $(8,14-16)$. The placenta itself is a major site for choline consumption and metabolism $(7,17)$. Given these facts, fetal choline concentrations could be a better reflection of fetal and placental choline distribution and metabolism than maternal choline concentrations.

Choline is the metabolic precursor of betaine. Betaine donates one-carbon group directly to homocysteine, generating dimethylglycine (DMG). In addition, metabolism of DMG generates two more one-carbon groups donated to folate. The exact role of betaine and DMG during pregnancy is not known, but an inverse relationship between betaine and homocysteine emphasizes the role of betaine in pregnancy $(18,19)$. Choline is essential for the developing and rapidly growing fetus. Maternal choline could possibly influence birth weight by changes in cell membrane synthesis, structural integrity and signaling, methylation reactions, or stem cell proliferation; fetal choline represents placental and fetal distribution and metabolism. Therefore, we hypothesized that lower choline concentrations would be associated with lower birth weight. We investigated this relationships between choline, betaine, and DMG concentrations and birth weight in both maternal and umbilical blood samples. 


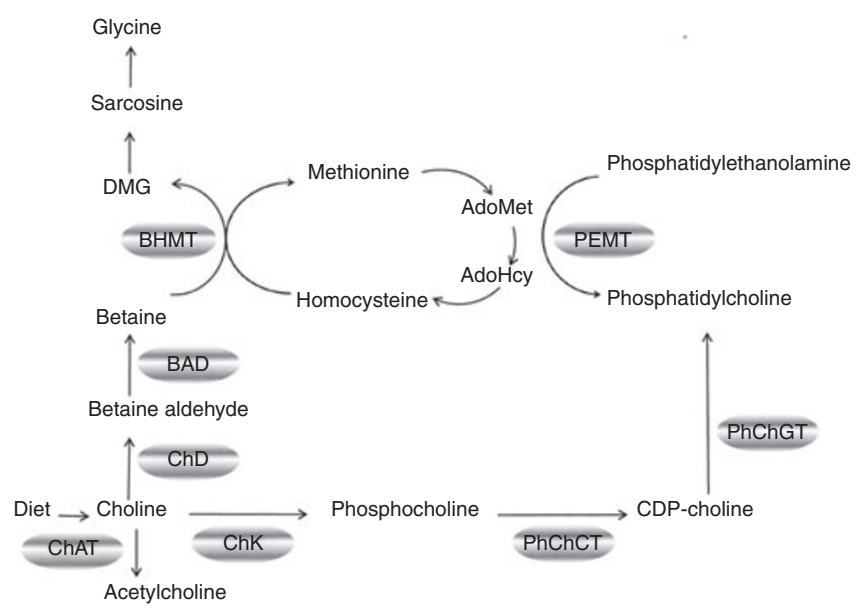

Figure 1. Choline metabolism. Choline is oxidized to betaine aldehyde, which is then converted in two steps to betaine. The first step is catalyzed by choline dehydrogenase $(\mathrm{ChD})$, the second step by betaine aldehyde dehydrogenase (BAD). Betaine can be used as a methyl donor in a remethylation reaction from homocysteine to methionine being catalyzed by betaine homocysteine methyltransferase (BHMT). This reaction generates dimethylglycine (DMG). Methionine is the precursor of S-adenosylmethionine, which serves as a methyl donor for numerous physiological reactions resulting in the formation of S-adenosylhomocysteine. Phosphorylation: choline can also be phosphorylated; choline kinase (ChK) catalyzes the reaction in which choline is phosphorylated to phosphocholine with adenosine triphosphate as the phosphate donor. The next step is the formation of cytidine diphosphate-choline (CDP-choline) from phosphocholine and cytidine triphosphate (CTP), which is catalyzed by CTP-phosphocholine cytidyl transferase (PhChCT). CDP-choline is subsequently combined with diacylglycerol to form phosphatidylcholine in a reaction catalyzed by phosphatidylcholine glyceride transferase (PhChGT). Acetylation: a small fraction of choline is acetylated; choline and acetyl coenzyme A form acetylcholine in a reaction that is catalyzed by choline acetyltransferase (ChAT). De novo synthesis of choline: sequential methylation of phosphatidylethanolamine, catalyzed by phosphatidylethanolamine- $N$-methyltransferase (PEMT), produces new choline molecules in the form of phosphatidylcholine (lecithin).

\section{RESULTS}

\section{Study Population}

Population characteristics are presented in Table 1. Most newborns were born at or near term (range $33+4$ to $43+2 \mathrm{wk}$ ), with only $4.9 \%$ of the newborns born before 37 gestational weeks and $6.6 \%$ after 42 gestational weeks. Birth weights ranged from 1,400 to $5,300 \mathrm{~g} ; 4.3 \%$ of the newborns from this study group were born with birth weight $\leq 2,500 \mathrm{~g}$. Four of 10 women gave birth for the first time. Male:female ratio of newborns was 55:45. Approximately 50\% used folic acid during pregnancy.

\section{Blood Indexes and Correlations}

Concentrations of umbilical and maternal choline, betaine, and DMG are shown in Table 2. The median concentration of choline in cord blood as compared with maternal blood was four times higher, whereas the betaine concentration was twice as high. The fetal-maternal gradient was less pronounced for DMG. All maternal and umbilical cord (UC) plasma concentrations were related. Correlations between umbilical and maternal analyte concentrations showed, as expected, correlations between choline and betaine in both maternal and UC plasma samples $(r=0.46$ and $r=0.32$, respectively, $P<0.001$ ).
Table 1. Population characteristics

\begin{tabular}{lc}
\hline & $N=1,126$ \\
\hline Age at delivery (years) & $31.9(22.9,41.0)$ \\
Gestational age (weeks) & $40.0(38.7,41.1)$ \\
Birth weight (grams) & $3,470(3,130,3,835)$ \\
Primiparity, $n / N(\%)$ & $457 / 1,105(41 \%)$ \\
Smoking, $n / N(\%)$ & $167 / 1,093(15 \%)$ \\
Use of folic acid, $n / N(\%)$ & $529 / 1,088(49 \%)$ \\
Male sex, $n / N(\%)$ & $501 / 1,122(45 \%)$ \\
\hline Results are expressed as median and interquartile range unless reported otherwise.
\end{tabular}

Table 2. Umbilical and maternal concentrations of choline and related methylamines

\begin{tabular}{lccc}
\hline & $\begin{array}{c}\text { Umbilical cord } \\
\text { plasma }^{\mathrm{a}, \mathrm{b}}\end{array}$ & Maternal plasma $^{\mathrm{a}}$ & Correlation $^{\mathrm{c}}$ \\
\cline { 2 - 4 } & $n=1,126$ & $n=366$ & $n=366$ \\
\hline Choline & $28.0(24.3,33.8)^{* *}$ & $7.0(6.1,8.3)$ & $0.11^{*}$ \\
Betaine & $24.6(20.8,29.5)^{* *}$ & $11.0(9.2,12.9)$ & $0.28^{* *}$ \\
DMG & $3.8(3.1,4.6)^{* *}$ & $2.1(1.7,2.5)$ & $0.39^{* *}$ \\
\hline
\end{tabular}

All concentrations are expressed as $\mu \mathrm{mol} / \mathrm{l}$.

DMG, dimethylglycine.

a Concentrations are expressed as median and interquartile range. ${ }^{b}$ All umbilical analytes are higher as compared with maternal analytes (paired samples $t$-test on logarithmically transformed data; $P<0.001$ ). ${ }^{c}$ Correlations are expressed as Pearson correlation coefficients after logarithmic transformation. ${ }^{*} P<0.05 .{ }^{* *} P<0.001$.

\section{Umbilical and Maternal Concentrations and Birth Weight}

Dose-response curves obtained by Gaussian generalized additive regression models show possible relationships between umbilical concentrations of choline, betaine, DMG, and birth weight (Figure 2). Generalized additive regression model curves concerning maternal concentrations did not reveal any relationship with birth weight (data not shown). Analyses of associations with birth weight are shown in Table 3. Smoking, gender, and parity were the strongest determinants of birth weight after gestational age (GA) in all the analyses. Higher umbilical choline and betaine concentrations were associated with lower birth weight, whereas higher umbilical DMG proved to be associated with higher birth weight. Maternal analytes were not associated with (standardized) birth weight in these analyses. Analysis of our data with restriction to a narrow GA span around term $(>39+0 ;<41+0$ wk of GA) showed similar results (data not shown).

\section{Associations With LBW}

Odds ratios for having a LBW newborn related to umbilical choline, betaine, and DMG concentrations are shown in Table 4. Concentrations of umbilical choline and betaine in the highest quartile were associated with an increased risk of having a LBW newborn, whereas maternal concentrations were not (data not shown). Calculation of odds ratios was considered not representative due to low numbers of LBW newborns in several groups.

\section{DISCUSSION}

We observed that umbilical choline and betaine concentrations were inversely associated with birth weight and DMG was positively associated with birth weight. To our best knowledge, this 

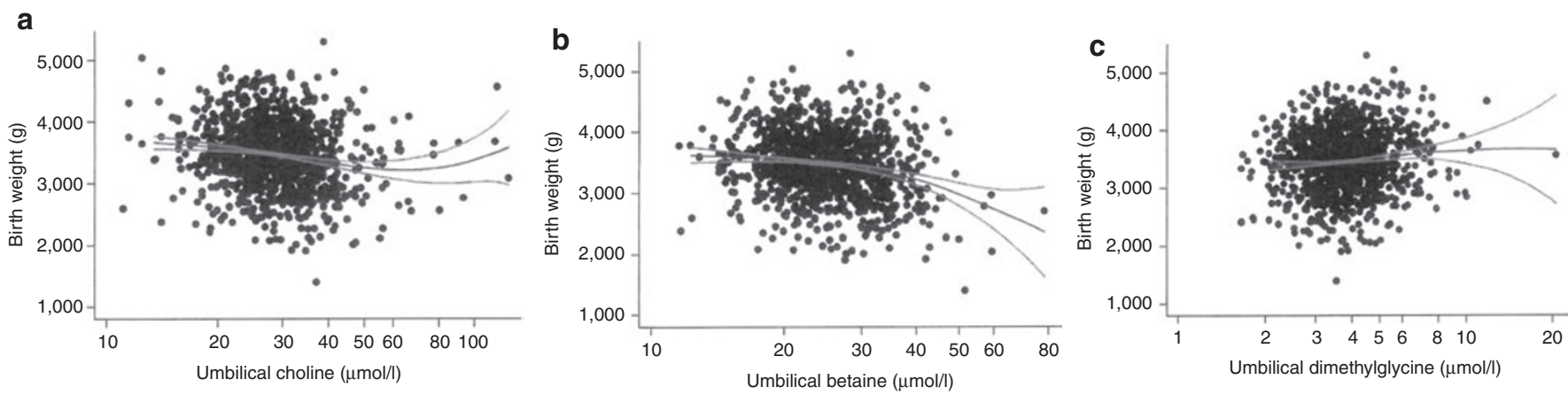

Figure 2. Gaussian generalized additive regression model curves showing nonlinear relationships between umbilical and maternal metabolites and birth weight. (a) Venous umbilical cord (UC) blood choline concentrations and birth weight. (b) Venous UC blood betaine concentrations and birth weight. (c) Venous UC blood dimethylglycine (DMG) concentrations and birth weight.

Table 3. Association between analytes and birth weight by linear regression

\begin{tabular}{|c|c|c|c|}
\hline & Model 1 & Model 2 & Model 3 \\
\hline & $\beta(95 \% \mathrm{Cl})$ & $\beta(95 \% \mathrm{Cl})$ & $\beta(95 \% \mathrm{Cl})$ \\
\hline $\begin{array}{l}\text { Gestational } \\
\text { age } \\
\text { (weeks) }\end{array}$ & $163(147,180)$ & - & - \\
\hline $\begin{array}{l}\text { Age at } \\
\text { delivery } \\
\text { (years) }\end{array}$ & $2.9(-4.2,9.9)$ & $3.5(-2.7,9.6)$ & $-8.2(-16.3,-0.2)$ \\
\hline Parity & $52(16,88)$ & $69(38,100)$ & $95.8(54.5,137)$ \\
\hline $\begin{array}{l}\text { Smoking } \\
\text { (no/yes) }\end{array}$ & $-276(-365,-187)$ & $-234(-312,-157)$ & $-251(-348,-153)$ \\
\hline $\begin{array}{l}\text { Use of } \\
\text { folic acid } \\
\text { (no/yes) }\end{array}$ & $15(-51,80)$ & $35(-22,92)$ & $38.2(-29.4,106)$ \\
\hline $\begin{array}{l}\text { Female } \\
\text { gender }\end{array}$ & $-135(-199,-71)$ & $-147(-202,-92)$ & $-156(-261,-51)$ \\
\hline \multicolumn{4}{|c|}{ UC blood plasma, $n=1,126$} \\
\hline $\begin{array}{l}\text { UC } \\
\text { choline }\end{array}$ & $-94(-128,-61)$ & $-55(-84,-26)$ & $-60(-89,-31)$ \\
\hline $\begin{array}{l}\text { UC } \\
\text { betaine }\end{array}$ & $-101(-133,-69)$ & $-57(-84,-29)$ & $-65(-94,-36)$ \\
\hline UCDMG & $52(20,85)$ & $38(9.6,66)$ & $35(6.1,63)$ \\
\hline \multicolumn{4}{|c|}{ Maternal plasma, $n=366$} \\
\hline $\begin{array}{l}\text { Maternal } \\
\text { choline }\end{array}$ & $41(-17,99)$ & $33(-19,85)$ & $27(-25,80)$ \\
\hline $\begin{array}{l}\text { Maternal } \\
\text { betaine }\end{array}$ & $12(-45,69)$ & $-9.9(-61,41)$ & $-16(-69,38)$ \\
\hline $\begin{array}{l}\text { Maternal } \\
\text { DMG }\end{array}$ & $-3.6(-63,55)$ & $-4.4(-58,49)$ & $-7.9(-62,46)$ \\
\hline
\end{tabular}

The coefficients presented give the change in birth weight (grams) for the several variables. Coefficients presented for plasma UC or maternal concentrations (choline, betaine, and DMG) are calculated using Z-scores of these metabolites. The coefficients represent the increase/decrease in birth weight (grams) following 1 SD increase in umbilical or maternal metabolites. All analyses were performed after logarithmic transformation. Model 1, univariate analysis of possible determinants on birth weight. Model 2, univariate analysis of possible determinants on standardized birth weights. Model 3, multivariate analysis of possible determinants on standardized birth weights. Model includes the specific metabolite and gestational age, age at delivery, parity, smoking, use of folic acid, and gender.

$\mathrm{Cl}$, confidence interval; DMG, dimethylglycine; $\mathrm{UC}$, umbilical cord; $\beta$, regression coefficient.
Table 4. ORs for having a newborn with standardized birth weight $\leq 2,500 \mathrm{~g}$ when umbilical metabolite concentrations are in the highest quartile

\begin{tabular}{lccc}
\hline$N=1,126$ & Choline & Betaine & DMG \\
\hline OR $(95 \% \mathrm{Cl})$ & $4.12(1.15,14.78)$ & $5.68(1.24,25.91)$ & $0.48(0.09,2.65)$ \\
AOR $(95 \% \mathrm{Cl})$ & $3.70(1.01,13.53)$ & $4.93(1.04,23.24)$ & $0.39(0.07,2.22)$ \\
\hline
\end{tabular}

Results are expressed as OR $(95 \% \mathrm{Cl})$.

AOR, OR adjusted for age at delivery, parity, smoking, use of folic acid, and newborn sex; $\mathrm{Cl}$, confidence interval; $\mathrm{DMG}$, dimethylglycine; OR, odds ratio.

is the largest research project to date studying the possible relationship between birth weight and choline and related methylamines in the mother as well as in the UC, which makes the results unique and robust. Venous UC blood has been drawn for practical reasons, but the correlation between concentration of metabolites in venous and arterial umbilical blood is high (20). We analyzed choline but also betaine and DMG, the important metabolites of choline oxidation pathway. This study was performed in newborns born at or near term and in an assumingly healthy pregnant population, which makes it possible to extrapolate these results to the general population. Adding mothernewborn pairs makes it possible to describe maternal-umbilical correlations for different metabolites and to study the effect of maternal analytes on birth weight as well. Unfortunately, other possible determinants of birth weight, such as maternal weight gain and BMI, are not known. Choline and betaine have been shown to have divergent associations with BMI in adults; by contrast, they have a similar direction of association in our study.

Maternal choline concentrations during pregnancy varying between 7.3 and $16.5 \mu \mathrm{mol} / \mathrm{l}$ have been reported by several authors $(8,14,15,18,20-22)$. Timing of maternal samples differed from $16 \mathrm{wk}$ of GA to delivery. Maternal choline, betaine, and DMG concentrations described in the literature are comparable with our results (18-22).

Several studies concerning umbilical choline concentrations have been performed $(8,15,20-23)$. They are small $(8,20,22,23)$, did not involve maternal and umbilical data (8), or did not evaluate the possible association between birth weight and choline concentrations $(8,15,21-23)$. UC plasma choline concentrations have been described between $\sim 25$ and $40 \mu \mathrm{mol} / \mathrm{l}(8,14,15,20,22,23)$, 


\section{Articles $\mid$ Hogeveen et al.}

which seems in line with our results. Betaine concentrations in UC blood vary from 21 to $29 \mu \mathrm{mol} / \mathrm{l}$, and DMG concentrations vary between 2 and $4 \mu \mathrm{mol} / \mathrm{l}$ (19-22), which is also in line with our results. To our knowledge, the influence of delivery mode and duration of labor on UC blood choline concentrations has not been studied. Unfortunately, no information on assisted vaginal delivery or duration of labor was recorded in our study.

The reported correlation coefficients of the relationship between maternal and venous UC metabolites vary from 0.06 to 0.26 for choline, 0.11 to 0.46 for betaine, and 0.58 to 0.73 for DMG (15,19-22). Furthermore, there are consistent reports on choline concentrations in cord plasma that are several fold higher than in maternal plasma, both in humans $(8,14,15,20-23)$ and animals (16). Similar results were obtained in the present study.

We found the well-known determinants for birth weight confirmed in our study: GA, newborn sex, smoking, and parity (24). Notably, higher UC plasma choline and betaine were related to lower birth weight in our study, whereas maternal metabolite concentrations were not associated with birth weight. Increased UC plasma DMG is associated with increased birth weight in this population, although this effect is small. The only study relating choline to birth weight was performed by Braekke et al. (20). They studied maternal, venous, and arterial UC blood concentrations of, among others, choline, betaine, and DMG. They did not reveal maternal choline as a determinant for infant birth weight percentile in 47 preeclamptic mothers compared with 51 controls (20).

In this population of well-nourished healthy mothers, birth weight showed a strong inverse relationship to umbilical choline and betaine, but not to maternal metabolites. Likewise, the previous study by Braekke et al. has demonstrated higher umbilical vein choline in 28 small-for-gestational-age compared with 15 non-small-for-gestational-age babies born to mothers with preeclampsia (20). Venous umbilical cord blood is a reflection of maternal concentrations, maternal-placental transfer and placental metabolism and reflects supply to the fetus. Arterial umbilical concentrations reflect fetal metabolism. Correlation between concentrations of metabolites in venous and arterial umbilical blood is high (20) and higher venous concentrations compared with arterial concentrations have been described suggesting uptake by the fetus $(15,20)$. High choline content in umbilical cord blood from small babies may reflect low choline consumption in fetal as well as in placental tissue, since the latter expresses enzymes involved in choline metabolism. If fetal consumption and metabolism are low, one would expect higher arterial concentrations and decreased venous-arterial differences in smaller babies compared with larger babies. This explanation could be supported by the observed positive relation between birth weight and umbilical DMG, which indicates that flux through the betaine-homocysteine methyltransferase pathway increases with increasing fetal size. DMG formation is probably confined to fetal tissues, because betaine-homocysteine methyltransferase is not expressed in human placental tissue (25). However, it cannot be ruled out that increased placental transport of choline triggered by slow fetal growth represents an alternative or complementary mechanism. Restriction analysis to near-term newborns showed similar results which suggests that the observed association between choline and related methylamines betaine and dimethylglycine and birth weight is not explained by GA.

Considering the positive effects of choline on the developing fetus in relation to brain development and memory, we certainly do not believe that a direct (toxic) effect of choline delivered to the fetus is likely to be the cause of the inverse relationship between umbilical cord plasma choline and birth weight in our study. This phenomenon might thus be a reflection of disturbances in placental choline metabolism. Decreased acetylation of choline could lead to higher free choline concentrations. The function of the high acetylcholine concentrations in the placenta are not known (26). Also, decreased choline kinase and/or cytidinetriphosphate-phosphocholine cytidyl transferase activity could lead to lower phosphatidylcholine concentrations. This is the predominant phospholipid in most mammalian membranes and important for the synthesis of lung surfactant, bile lipids, and plasma lipoproteins. Unfortunately, we were not able to measure acetylcholine or phosphatidylcholine in our samples. The placenta is a major site for choline utilization; hence, higher venous umbilical cord plasma choline concentrations could reflect a disturbed placental function, eventually leading to disturbed fetal growth and lower birth weight. Cellular degradation due to placental damage, e.g., due to hypoxia, leads to release of choline from membrane phosphatidylcholine or acetylcholine into umbilical circulation and will increase umbilical venous choline concentrations. The fact that higher choline and betaine but lower DMG concentrations are associated with lower birth weight could also indicate decreased flux through the choline oxidation pathway. DMG plays an important role in generating glycine, an amino acid involved in synthesis of purines, creatine, collagen, elastin, and glutathione. Glutathione is involved in the protection against oxidative stress and regulation of protein and DNA synthesis.

In our study consisting of 1,126 cord blood samples, birth weight showed a strong inverse relationship to umbilical choline and betaine. These results might reflect a disturbed placental function where choline serves as a marker. Further research is needed to clarify these findings and to study if we can influence these pathways in such a way that the risk of low birth weight will decrease. Determination of total choline and the different forms of choline in maternal blood and also both arterial and venous umbilical cord blood in normal pregnancies together with that of pregnancies complicated by intrauterine growth retardation, placental dysfunction or low birth weight should be involved in such research.

\section{METHODS}

\section{Participants}

Patients were recruited as described before (27). In summary, patients were included in our study when visiting the outpatient clinic and/ or the delivery ward of the department of Obstetrics and Gynecology at the Canisius Wilhelmina Hospital in Nijmegen, the Netherlands. All pregnant women attending the department were potentially eligible. Women attending the outpatient clinic or the delivery ward were asked to participate when the time schedule of the clinician, the nurses, and/ or the administrative worker allowed this. All women gave informed consent. Maternal blood samples $(n=366)$ were collected between 30 
and $34 \mathrm{wk}$ of GA during routine blood sampling from women visiting the outpatient department of the hospital during pregnancy, and $366 \mathrm{UC}$ blood samples were collected from their newborns at delivery. In addition, from the participants only seen in the delivery ward, 760 UC blood samples were collected. Approximately $20 \%$ of women delivering in this hospital during the study period (2002-2004) were included. To check whether the included women were representative of the entire population visiting this hospital, we randomly selected 500 additional women from the same period from the delivery ward records and found comparable results for maternal age, GA, birth weight, and newborn sex (data not shown). We excluded twin pregnancies. All mothers were asked to fill out a questionnaire at the time of blood sampling. Informed consent was obtained from all participants, and the institutional review board of the Radboud University Nijmegen Medical Center approved our study protocol.

\section{Data Collection}

Birth weight and GA were obtained from the delivery charts. Information on parity, medical history, education, smoking during pregnancy, and use of folic acid was collected through questionnaires and, if necessary, review of patient charts.

\section{Blood Sampling}

Immediately after birth, the UC was clamped, and venous cord blood was collected into a $3.5 \mathrm{ml}$ EDTA container and immediately placed on ice. Maternal venous blood was drawn between 30 and 34 wk of GA and collected in $3.5 \mathrm{ml}$ EDTA containers and immediately placed on ice. After centrifugation (within $2 \mathrm{~h}$ after sampling), plasma was separated and EDTA plasma and blood cells were stored at $-20^{\circ} \mathrm{C}$ until analyzed.

\section{Biochemical Analyses}

All analytes were determined in both maternal and UC EDTA plasma prepared from venous blood. Plasma choline, betaine, and DMG were determined as described before (28).

\section{Statistical Analyses}

Results are presented as median values with the interquartile range. Variables were log-transformed before further analysis. Maternal and umbilical samples were compared using paired samples $t$-test. Correlations between variables were estimated as Pearson correlations on the transformed data and $Z$-scores were calculated. Linear regression analysis was used to determine associations with of birth weight. Because birth weight and GA were strongly related, we calculated birth weights standardized for GA using linear regression on birth weight and GA. Again, $Z$-scores were calculated and linear regression analyses were performed using the standardized birth weight as dependent variable. To show nonlinear relationship, dose-response curves were constructed to describe correlations between umbilical and maternal concentrations and birth weight, using generalized additive regression models (29). Logistic regression analysis was used to determine odds ratios for umbilical and maternal analytes in relation to birth weight. LBW was defined as standardized birth weight $\leq 2,500 \mathrm{~g}$. STATA for Windows version 11.0 (STATA, College Station, TX) was used for all statistical analyses.

\section{ACKNOWLEDGMENTS}

We thank Gry Kvalheim and Arno van Rooij for their expert technical assistance contributing to the laboratory results.

\section{STATEMENT OF FINANCIAL SUPPORT}

No financial assistance was received to support this study.

\section{REFERENCES}

1. Papageorgou A, Pelausa E, Kovacs L. The extremely low birth weight infant. In: MacDonald MG, Seshia MMK, eds. Avery's Neonatology, 5th edn. Philadelphia, PA: Lippincott Williams \& Wilkins, 2009:978-1025.

2. Barker DJ. The fetal and infant origins of disease. Eur J Clin Invest 1995;25:457-63.

3. Buchman AL, Sohel M, Brown M, et al. Verbal and visual memory improve after choline supplementation in long-term total parenteral nutrition: a pilot study. JPEN J Parenter Enteral Nutr 2001;25:30-5.

4. Zeisel SH. Choline, homocysteine, and pregnancy. Am J Clin Nutr 2005;82:719-20.
5. Zeisel SH, Mar MH, Zhou Z, da Costa KA. Pregnancy and lactation are associated with diminished concentrations of choline and its metabolites in rat liver. J Nutr 1995;125:3049-54.

6. Zeisel SH. Choline: critical role during fetal development and dietary requirements in adults. Annu Rev Nutr 2006;26:229-50.

7. Sweiry JH, Page KR, Dacke CG, Abramovich DR, Yudilevich DL. Evidence of saturable uptake mechanisms at maternal and fetal sides of the perfused human placenta by rapid paired-tracer dilution: studies with calcium and choline. J Dev Physiol 1986;8:435-45.

8. McMahon KE, Farrell PM. Measurement of free choline concentrations in maternal and neonatal blood by micropyrolysis gas chromatography. Clin Chim Acta 1985;149:1-12.

9. Craciunescu CN, Wu R, Zeisel SH. Diethanolamine alters neurogenesis and induces apoptosis in fetal mouse hippocampus. FASEB J 2006;20: $1635-40$.

10. Zeisel SH, Niculescu MD. Perinatal choline influences brain structure and function. Nutr Rev 2006;64:197-203.

11. Thomas JD, Garrison M, O'Neill TM. Perinatal choline supplementation attenuates behavioral alterations associated with neonatal alcohol exposure in rats. Neurotoxicol Teratol 2004;26:35-45.

12. Shaw GM, Finnell RH, Blom HJ, et al. Choline and risk of neural tube defects in a folate-fortified population. Epidemiology 2009;20:714-9.

13. Shaw GM, Carmichael SL, Yang W, Selvin S, Schaffer DM. Periconceptional dietary intake of choline and betaine and neural tube defects in offspring. Am J Epidemiol 2004;160:102-9.

14. Ozarda Ilcol Y, Uncu G, Ulus IH. Free and phospholipid-bound choline concentrations in serum during pregnancy, after delivery and in newborns. Arch Physiol Biochem 2002;110:393-9.

15. Buchman AL, Sohel M, Moukarzel A, et al. Plasma choline in normal newborns, infants, toddlers, and in very-low-birth-weight neonates requiring total parenteral nutrition. Nutrition 2001;17:18-21.

16. Zeisel SH, Epstein MF, Wurtman RJ. Elevated choline concentration in neonatal plasma. Life Sci 1980;26:1827-31.

17. Yudilevich DL, Sweiry JH. Membrane carriers and receptors at maternal and fetal sides of the placenta by single circulation paired-tracer dilution: evidence for a choline transport system. Contrib Gynecol Obstet 1985;13:158-61.

18. Velzing-Aarts FV, Holm PI, Fokkema MR, van der Dijs FP, Ueland PM, Muskiet FA. Plasma choline and betaine and their relation to plasma homocysteine in normal pregnancy. Am J Clin Nutr 2005;81:1383-9.

19. Wallace JM, Bonham MP, Strain J, et al. Homocysteine concentration, related B vitamins, and betaine in pregnant women recruited to the Seychelles Child Development Study. Am J Clin Nutr 2008;87:391-7.

20. Braekke K, Ueland PM, Harsem NK, Karlsen A, Blomhoff R, Staff AC. Homocysteine, cysteine, and related metabolites in maternal and fetal plasma in preeclampsia. Pediatr Res 2007;62:319-24.

21. Molloy AM, Mills JL, Cox C, et al. Choline and homocysteine interrelations in umbilical cord and maternal plasma at delivery. Am J Clin Nutr 2005;82:836-42.

22. Friesen RW, Novak EM, Hasman D, Innis SM. Relationship of dimethylglycine, choline, and betaine with oxoproline in plasma of pregnant women and their newborn infants. J Nutr 2007;137:2641-6.

23. Ilcol YO, Ozbek R, Hamurtekin E, Ulus IH. Choline status in newborns, infants, children, breast-feeding women, breast-fed infants and human breast milk. J Nutr Biochem 2005;16:489-99.

24. Cogswell ME, Yip R. The influence of fetal and maternal factors on the distribution of birthweight. Semin Perinatol 1995;19:222-40.

25. Solanky N, Requena Jimenez A, D’Souza SW, Sibley CP, Glazier JD. Expression of folate transporters in human placenta and implications for homocysteine metabolism. Placenta 2010;31:134-43.

26. Zeisel SH. Dietary choline: biochemistry, physiology, and pharmacology. Annu Rev Nutr 1981;1:95-121.

27. Hogeveen M, Blom HJ, van der Heijden EH, et al. Maternal homocysteine and related B vitamins as risk factors for low birthweight. Am J Obstet Gynecol 2010;202:572.e1-6.

28. Ueland PM, Midttun O, Windelberg A, Svardal A, Skålevik R, Hustad S. Quantitative profiling of folate and one-carbon metabolism in largescale epidemiological studies by mass spectrometry. Clin Chem Lab Med 2007;45:1737-45.

29. Hastie T, Tibshirani R. Generalized additive models for medical research Stat Methods Med Res 1995;4:187-96. 